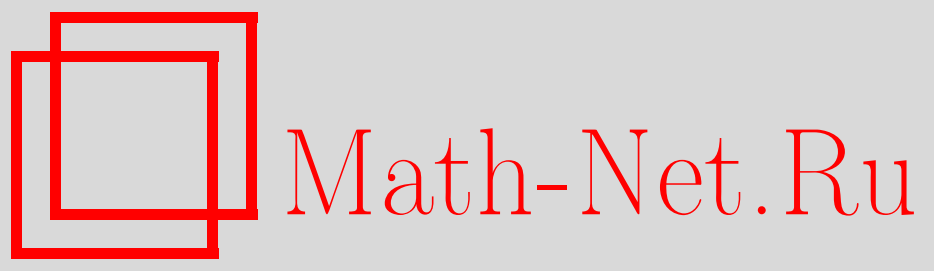

Дж. Харнад, Й. В. ван де Лёр, А. Ю. Орлов, Кратные суммы и интегралы как тау-функции нейтральной иерархии Кадомцева-Петвиашвили, ТМФ, 2011, том 168, номер 1, 112-124

DOI: https://doi.org/10.4213/tmf6667

Использование Общероссийского математического портала Math-Net.Ru подразумевает, что вы прочитали и согласны с пользовательским соглашением http://www.mathnet.ru/rus/agreement

Параметры загрузки:

IP : 3.81 .55 .215

26 апреля 2023 г., 18:36:40

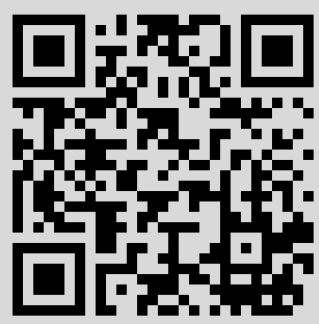




\title{
КРАТНЫЕ СУММЫ И ИНТЕГРАЛЫ КАК ТАУ-ФУНКЦИИ НЕЙТРАЛЬНОЙ ИЕРАРХИИ КАДОМЦЕВА-ПЕТВИАШВИЛИ
}

\begin{abstract}
Кратные суммы и кратные интегралы рассмотрены как тау-функции так называемой нейтральной иерархии Кадомцева-Петвиашвили на решетке корней типа В; для их вывода в качестве простейшего средства использованы нейтральные фермионы. Суммы берутся по проективным функциям Шура $Q_{\alpha}$ для строгих разбиений $\alpha$. Рассмотрено два типа таких сумм: взвешенные суммы $Q_{\alpha}$ по строгим разбиениям $\alpha$ и суммы по произведениям $Q_{\alpha} Q_{\gamma}$. Таким способом получаются дискретные аналоги бета-ансамблей $(\beta=1,2,4)$. Непрерывные аналоги представлены в виде кратных интегралов, которые интересны с точки зрения их применения в ряде задач математики и физики.
\end{abstract}

Ключевые слова: интегрируемые системы, симметрические функции, проективные функции Шура, случайные разбиения.

\section{1. ВВЕДЕНИЕ}

В настоящей работе рассматриваются кратные суммы и кратные интегралы, представляющие интерес с точки зрения некоторых задач математики и физики. Кратные суммы появляются в моделях случайных разбиений (как было показано в обзорной работе [1]) и случайных движений частиц [2] (см. также работу [3]). Кратные интегралы дают некоторые аналоги бета-ансамблей случайных матриц, $\beta=1,2,4$, а также аналог двухматричных моделей [4]. Мы рассматриваем деформации меры, определяемые в терминах четырех полубесконечных наборов параметров, и связываем производящую функцию $Z$ со сдвоенной и двухкомпонентной иерархией Кадомцева-Петвиашвили на решетке корней типа В (иерархией 2B-KП). Основной прием заключается в использовании одно- и двухкомпонентных нейтральных фермионов, которые обеспечивают связь с интегрируемыми системами, известными

${ }^{*}$ Centre de recherches mathématiques, Université de Montréal, Montréal, Canada. E-mail: harnad@crm.umontreal.ca

${ }^{\dagger}$ Department of Mathematics and Statistics, Concordia University, Montréal, Canada

${ }^{\ddagger}$ Mathematical Institute, University of Utrecht, Utrecht, The Netherlands.

E-mail: J.W.vandeLeur@uu.nl

${ }^{\S}$ Институт океанологии, Москва, Россия. E-mail: orlovs55@mail.ru 
как нейтральные иерархии В-КП [5], [6]. Одна из версий таких иерархий была впервые введена в работах [5], [6], в настоящей работе мы используем другую версию, введенную в работе [7].

\section{2. СУММЫ ПО ПРОЕКТИВНЫМИ ФУНКЦИЯМИ ШУРА}

Мы рассматриваем суммы по строгим разбиениям, разбиения будем обозначать греческими буквами $\alpha, \beta$. Напомним [8], что строгое разбиение $\alpha$ представляет собой набор целых чисел (частей) $\left(\alpha_{1}, \ldots, \alpha_{k}\right)$ при $\alpha_{1}>\cdots>\alpha_{k} \geqslant 0$. Длина разбиения $\alpha$, обозначаемая через $\ell(\alpha)$, равна числу ненулевых частей, таким образом, в данном случае $\ell(\alpha)=k$ или $\ell(\alpha)=k-1$. Пусть $D P$ - множество строгих разбиений (т. е. разбиений с различными частями). Нам также потребуется подмножество множества $D P$, которое мы обозначим как $D P^{2}$, состоящее из всех разбиений вида $\left(\alpha_{1}, \alpha_{1}-1, \alpha_{3}, \alpha_{3}-1, \ldots, \alpha_{k-1}, \alpha_{k-1}-1\right)$. Рассмотрим следующие суммы (при $\left.L \in \mathbb{N}^{+}, t:=\left(t_{1}, t_{3}, \ldots\right), \mathbf{t}^{*}:=\left(t_{1}^{*}, t_{3}^{*}, \ldots\right), \overline{\mathbf{t}}:=\left(\bar{t}_{1}, \bar{t}_{3}, \ldots\right)\right)$ :

$$
\begin{aligned}
& S_{0}(\mathbf{t}, L):=\sum_{\substack{\alpha \in D P \\
\alpha_{1} \leqslant L}} Q_{\alpha}\left(\frac{\mathbf{t}}{2}\right), \\
& S_{1}\left(\mathbf{t}, \mathbf{t}^{*}\right):=\sum_{\alpha \in D P} e^{-U_{\alpha}\left(\mathbf{t}^{*}\right)} Q_{\alpha}\left(\frac{\mathbf{t}}{2}\right), \\
& S_{2}\left(\mathbf{t}, \overline{\mathbf{t}}, \mathbf{t}^{*}\right):=\sum_{\alpha \in D P} e^{-U_{\alpha}\left(\mathbf{t}^{*}\right)} Q_{\alpha}\left(\frac{\mathbf{t}}{2}\right) Q_{\alpha}\left(\frac{\overline{\mathbf{t}}}{2}\right), \\
& S_{00}(\mathbf{t}, \overline{\mathbf{t}}, L):=\sum_{\substack{\alpha \in D P \\
\alpha_{1} \leqslant L}} Q_{\alpha}\left(\frac{\mathbf{t}}{2}\right) Q_{\alpha}\left(\frac{\overline{\mathbf{t}}}{2}\right), \\
& S_{3}\left(\mathbf{t}, A^{\mathrm{c}}\right):=\sum_{\alpha \in D P} A_{\alpha}^{\mathrm{c}} Q_{\alpha}\left(\frac{\mathbf{t}}{2}\right), \\
& S_{4}\left(\mathbf{t}, \mathbf{t}^{*}\right):=\sum_{\alpha \in D P^{2}} e^{-U_{\alpha}\left(\mathbf{t}^{*}\right)} Q_{\alpha}\left(\frac{\mathbf{t}}{2}\right), \\
& S_{5}(\mathbf{t}, \overline{\mathbf{t}}, D):=\sum_{\substack{\alpha, \beta \in D P, \ell(\alpha)=\ell(\beta)}} Q_{\alpha}\left(\frac{\mathbf{t}}{2}\right) D_{\alpha, \beta} Q_{\beta}\left(\frac{\overline{\mathbf{t}}}{2}\right) .
\end{aligned}
$$

Здесь проективные функиии Шура $Q_{\alpha}$ являются взвешенными многочленами по переменным $\mathbf{t}=\left(t_{1}, t_{3}, t_{5}, \ldots\right), \operatorname{deg} t_{m}=m$, занумерованными строгими разбиениями (их подробное определение см. в работе [8]). Известно, что каждая функция $Q_{\alpha}(\mathbf{t} / 2)$ представляет собой тау-функцию иерархии В-КП [5], [6]. Это изящное наблюдение было сделано в работах [9], [10]. Тот факт, что в высших временах $t_{2 m-1}$ иерархии В-КП появляются только нечетные нижние индексы, связан с тем, что она получается редукцией из иерархии КП. Коэффициенты $U_{\alpha}$ определяются как

$$
U_{\alpha}:=\sum_{i=1}^{k} U_{\alpha_{i}}, \quad U_{n}:=U_{n}^{(0)}-\sum_{\substack{m \neq 0, n \text { нечетное }}} n^{m} t_{m}^{*}-\ln n !, \quad n \in \mathbb{N}^{+},
$$

для некоторого набора постоянных $\left\{U_{n}^{(0)}\right\}$. 
Коэффициенты $A_{\alpha}^{\mathrm{c}}$ в правой части (5) определены в терминах пар $(A, a)$, где $A$ - бесконечная кососимметричная матрица, $a$ - бесконечный вектор. Для строгого разбиения $\alpha=\left(\alpha_{1}, \ldots, \alpha_{k}\right)$, где $\alpha_{k}>0$, число $A_{\alpha}^{\text {c } о п р е д е л я е т с я ~ к а к ~ п ф а ф ф и а н ~}$ антисимметричной $(2 n \times 2 n)$-матрицы $\tilde{A}$,

$$
A_{\alpha}^{\mathrm{c}}:=\operatorname{Pf}[\tilde{A}],
$$

где для четных $k=2 n$

$$
\tilde{A}_{i j}=-\tilde{A}_{j i}:=A_{\alpha_{i}, \alpha_{j}}, \quad 1 \leqslant i<j \leqslant 2 n,
$$

а для нечетных $k=2 n-1$

$$
\tilde{A}_{i j}=-\tilde{A}_{j i}:= \begin{cases}A_{\alpha_{i}, \alpha_{j}}, & \text { если } 1 \leqslant i<j \leqslant 2 n-1, \\ a_{\alpha_{i}}, & \text { если } 1 \leqslant i<j=2 n .\end{cases}
$$

Кроме того, мы полагаем $A_{0}^{\mathrm{c}}=1$.

Коэффициенты $D_{\alpha, \beta}$ в $(7)$ определяются как $D_{\alpha, \beta}=\operatorname{det}\left(D_{\alpha_{i}, \beta_{j}}\right)$, где $D$ - заданная постоянная бесконечная матрица.

Замечание 1. Ряды (1)-(4) могут быть получены путем специализации $A^{\mathrm{c}}$ в ряде (5). Если положить

$$
A_{n m}=\frac{1}{2} e^{-U_{m}-U_{n}} \operatorname{sgn}(n-m), \quad a_{n}=e^{-U_{n}},
$$

то получим (2). Если далее выбрать $U_{n}=0$ и $U_{n}=+\infty$ для $n \leqslant L$ и $n>L$ соответственно, то получим (1). Если положить

$$
A_{n m}=\frac{1}{2} e^{-U_{m}-U_{n}} Q_{(n, m)}\left(\frac{\overline{\mathbf{t}}}{2}\right), \quad a_{n}=e^{-U_{n}} Q_{(n)}\left(\frac{\overline{\mathbf{t}}}{2}\right),
$$

то получим (3). Снова выбирая $U_{n}=0$ и $U_{n}=+\infty$ для $n \leqslant L$ и $n>L$ соответственно, получаем (4). Ряд (6) получается из (5), если взять $A_{n m}=\delta_{n+1, m}-\delta_{m+1, n}$. Суммы (4) и (3) также можно получить как частные случаи (7): если положить $D_{n m}=e^{-U_{m}-U_{n}} \delta_{n, m}$, то мы получаем (3).

Все эти суммы представляют собой частные примеры тау-функций иерархии В-КП, введенных в работе [5] и определяющих решения того, что в работе [7] было названо нейтральной иерархией В-КП. Их можно далее конкретизировать, если выбрать $\mathbf{t}=\mathbf{t}_{\infty}:=(1,0,0, \ldots)$. Тогда

$$
Q_{\alpha}\left(\frac{\mathbf{t}_{\infty}}{2}\right)=\Delta^{*}(\alpha) \prod_{i=1}^{k} \frac{1}{\alpha_{i} !}, \quad \alpha=\left(\alpha_{1}, \ldots, \alpha_{k}\right),
$$

где

$$
\Delta^{*}(\alpha)=\Delta_{k}^{*}(\alpha):=\prod_{0<i<j \leqslant k} \frac{\alpha_{i}-\alpha_{j}}{\alpha_{i}+\alpha_{j}}
$$


Для этого случая получаем

$$
\begin{aligned}
S_{1}\left(\mathbf{t}_{\infty}, \mathbf{t}^{*}\right) & =\sum_{\alpha \in D P} \Delta^{*}(\alpha) \prod_{i=1}^{k} \frac{e^{-U_{\alpha_{i}}\left(\mathbf{t}^{*}\right)}}{\alpha_{i} !}, \\
S_{2}\left(\mathbf{t}_{\infty}, \mathbf{t}_{\infty}, \mathbf{t}^{*}\right) & =\sum_{\alpha \in D P}\left(\Delta^{*}(\alpha)\right)^{2} \prod_{i=1}^{k} \frac{e^{-U_{\alpha_{i}}\left(\mathbf{t}^{*}\right)}}{\left(\alpha_{i} !\right)^{2}}, \\
S_{4}\left(\mathbf{t}_{\infty}, \mathbf{t}^{*}\right) & =\sum_{\alpha \in D P^{\prime}}\left(\widetilde{\Delta}^{*}(\alpha)\right)^{4} \prod_{i=1}^{k} \frac{e^{-U_{\alpha_{i}}\left(\mathbf{t}^{*}\right)-U_{\alpha_{i}+1}\left(\mathbf{t}^{*}\right)}}{\alpha_{i} !\left(\alpha_{i}+1\right) !}, \\
S_{5}\left(\mathbf{t}_{\infty}, \mathbf{t}_{\infty}, \mathbf{t}^{*}\right) & =\sum_{k=0}^{\infty} \frac{1}{k !} \sum_{\substack{\alpha, \beta \in D P, \ell(\alpha)=\ell(\beta)=k}}^{k} \Delta^{*}(\alpha) \Delta^{*}(\beta) \prod_{i=1}^{k} \frac{D_{\alpha_{i}, \beta_{i}}}{\alpha_{i} ! \beta_{i} !} .
\end{aligned}
$$

Здесь $D P^{\prime}$ - множество всех строгих разбиений $\left(\alpha_{1}, \alpha_{2}, \ldots, \alpha_{N}\right), \alpha_{N}>0$, в которых $\alpha_{i}>\alpha_{i+1}+1, i=1, \ldots, N-1$, и

$$
\left(\widetilde{\Delta}^{*}(\alpha)\right)^{4}:=\prod_{i<j \leqslant N} \frac{\left(\alpha_{i}-\alpha_{j}\right)^{2}\left(\left(\alpha_{i}-\alpha_{j}\right)^{2}-1\right)}{\left(\alpha_{i}+\alpha_{j}\right)^{2}\left(\left(\alpha_{i}+\alpha_{j}\right)^{2}-1\right)} .
$$

Если в приведенных выше выражениях заменить $\Delta^{*}$ на определитель Вандермонда $\Delta$, то в результате полученные суммы (11) можно рассматривать как дискретные аналоги матричных моделей (см. работу [11]).

2.1. Примеры. Приведем несколько примеров применения выписанных выше сумм.

Пример 1. Сумма (4) впервые была рассмотрена в работе [12] при исследовании сдвинутой меры Шура.

Пример 2. Суммы (2) и (3) можно рассматривать как обобщения гипергеометрических функций на случай многих переменных. Например, обобщение гипергеометрической функции типа ${ }_{p} F_{r}$ можно получить из выражений $(2)$ и $(3)$, если определить параметры $U_{n}$ в терминах гамма-функций,

$$
U_{n}=\ln \frac{\prod_{i=1}^{p} \Gamma\left(n+a_{i}\right)}{\prod_{i=1}^{r} \Gamma\left(n+b_{i}\right)},
$$

для некоторого набора $p+r$ постоянных $\left\{a_{i}, b_{i}\right\}$. Суммы типа (3) были рассмотрены в работе [13], а суммы типа (2) являются новыми (подробному рассмотрению последнего случая будет посвящена отдельная работа). Можно показать, что и ряд (2), и ряд (3) записываются как пфаффианы матриц, элементы которых выражаются через функции ${ }_{p} F_{r}$ и обладают многими свойствами обычных гипергеометрических функций ${ }_{p} F_{r}$. Аналоги базисных ( $q$-деформированных) гипергеометрических функций можно получить схожим образом.

ПримеР 3. Рассмотрим модели случайных строгих разбиений $\alpha$, где относительный вес $W_{\alpha}$ определяется одним из следующих выражений:

$$
W_{\alpha}=A_{\alpha}^{\mathrm{c}} Q_{\alpha}\left(\frac{\mathbf{t}}{2}\right)
$$


где $A^{\mathrm{c}}=(A, a)$ и $\mathbf{t}=\left(t_{1}, t_{3}, \ldots\right)$ - параметры модели; или

$$
W_{\alpha}=e^{-U_{\alpha}\left(\mathbf{t}^{*}\right)} Q_{\alpha}\left(\frac{\mathbf{t}}{2}\right),
$$

где параметры имеют вид $\mathbf{t}=\left(t_{1}, t_{3}, \ldots\right)$ и $U=\left(U_{0}, U_{1}, \ldots\right)$; или

$$
W_{\alpha}=e^{-U_{\alpha}\left(\mathbf{t}^{*}\right)} Q_{\alpha}\left(\frac{\mathbf{t}}{2}\right) Q_{\alpha}\left(\frac{\overline{\mathbf{t}}}{2}\right),
$$

где $\mathbf{t}=\left(t_{1}, t_{3}, \ldots\right), \overline{\mathbf{t}}=\left(\bar{t}_{1}, \bar{t}_{3}, \ldots\right)$ и $U=\left(U_{0}, U_{1}, \ldots\right)$ - независимые параметры. Заметим, что модели (13) и (14) являются частными случаями модели (12). Ряды $S_{3}, S_{1}$ и $S_{2}$ можно рассматривать как нормировочные множители (статистические суммы) соответственно для моделей (12), (13) и (14). Аналогично ряд $S_{5}$ представляет собой статистическую сумму для модели строгих биразбиений.

Пример 4. Ряды $S_{1}$ и $S_{2}$ использовались в работе [14], где рассматривались случайные осциллирующие диаграммы Юнга, связанные со строгими разбиениями.

ЗАмЕчАниЕ 2. Фермионное представление этих моделей позволяет вычислить их корреляционные функции стандартным способом (см., например, работы [15]-[18]).

2.2. Нейтральные фермионы. Чтобы построить тау-функции иерархии В-КП и двухкомпонентной иерархии В-КП [6], [7], а также тау-функции их сдвоенных версий (2В-КП), которые мы вводим ниже, нам потребуются нейтралъные свободные

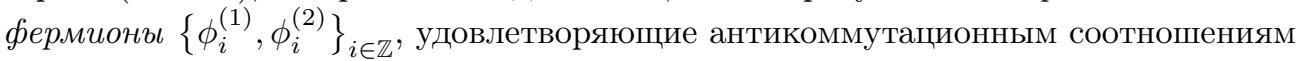

$$
\left[\phi_{n}^{(a)}, \phi_{m}^{(b)}\right]_{+}=(-1)^{n} \delta_{n,-m} \delta_{a, b}, \quad a, b=1,2 .
$$

Для однокомпонентых иерархий В-КП и 2В-КП используется только первая компонента $\phi_{n}:=\phi_{n}^{(1)}, n \in \mathbb{Z}$. Выберем фермионное пространство Фока, как в работе [7] (в отличие от работы [6]). А именно, действие нейтральных фермионов на вакуумные состояния определим формулами

$$
\begin{aligned}
\phi_{n}^{(a)}|0\rangle & =0, & \langle 0| \phi_{-n}^{(a)} & =0, \quad n<0, \\
\phi_{0}^{(a)}|0\rangle & =\frac{1}{\sqrt{2}}|0\rangle, & \langle 0| \phi_{0}^{(a)} & =\frac{1}{\sqrt{2}}\langle 0|,
\end{aligned}
$$

где соотношения (16) выбраны в соответствии с работой [7] (некоторые подробности связи между работами [6] и [7] можно также найти в приложении А.1 к версии работы [14], находящейся в электронном архиве).

Для линейных комбинаций $w_{k}=\sum_{a} \sum_{n} c_{k, n}^{(a)} \phi_{n}^{(a)}, k=1,2, \ldots$, из теоремы Вика следует, что для произвольных произведений четного числа полей $w_{k}$

$$
\begin{aligned}
\left\langle w_{1} w_{2} \ldots w_{2 n-1} w_{2 n}\right\rangle & =\sum_{\sigma \in S_{2 n}} \operatorname{sgn}(\sigma)\left\langle w_{\sigma(1)} w_{\sigma(2)}\right\rangle \ldots\left\langle w_{\sigma(2 n-1)} w_{\sigma(2 n)}\right\rangle=: \\
& =: \operatorname{Pf}\left(\left(\left\langle w_{i} w_{j}\right\rangle\right)_{1 \leqslant i, j \leqslant 2 n}\right),
\end{aligned}
$$

где сумма берется по всем перестановкам из группы перестановок $S_{2 n}$. 
Нам также потребуются фермионные поля

$$
\phi^{(a)}(z)=\sum_{n \in \mathbb{Z}} \phi_{n}^{(a)} z^{n}, \quad a=1,2 .
$$

Для вычисления интегралов воспользуемся формулой

$$
\left\langle 0\left|\phi^{(b)}\left(z_{1}\right) \phi^{(a)}\left(z_{2}\right)\right| 0\right\rangle=\frac{1}{2}\left(\frac{z_{1}-z_{2}}{z_{1}+z_{2}}\right) \delta_{a b} .
$$

Для $m \in \mathbb{N}^{+}$из теоремы Вика следует, что

$$
\left\langle 0\left|\phi^{(a)}\left(z_{1}\right) \phi^{(a)}\left(z_{2}\right) \ldots \phi^{(a)}\left(z_{m}\right)\right| 0\right\rangle=\left(\frac{1}{2}\right)^{m / 2} \Delta_{m}^{*}(z) .
$$

Заметим, что вследствие соотношений (16) это вакуумное среднее отлично от нуля при нечетных $m$.

2.3. Тау-функции иерархии В-КП. Тау-функция сдвоенной двухкомпонентной иерархии 2В-КП может быть определена как

$$
\begin{aligned}
& \tau^{2 \mathrm{c}-2 \mathrm{~B}-\mathrm{K} \Pi}\left(\mathbf{t}^{(1)}, \mathbf{t}^{(2)}, \overline{\mathbf{t}}^{(1)}, \overline{\mathbf{t}}^{(2)}, A\right)= \\
& \quad=\left\langle 0\left|\Gamma^{(1)}\left(\mathbf{t}^{(1)}\right) \Gamma^{(2)}\left(\mathbf{t}^{(2)}\right) \exp \left\{\sum_{a, b} \sum_{n, m} A_{n m}^{a, b} \phi_{n}^{(a)} \phi_{m}^{(b)}\right\} \bar{\Gamma}^{(1)}\left(\overline{\mathbf{t}}^{(1)}\right) \bar{\Gamma}^{(2)}\left(\overline{\mathbf{t}}^{(2)}\right)\right| 0\right\rangle,
\end{aligned}
$$

где

$$
\begin{gathered}
\Gamma^{(a)}\left(\mathbf{t}^{(a)}\right):=\exp \left\{\sum_{\substack{n \geqslant 1, n \text { нечетне }}} B_{n}^{a} t_{n}^{(a)}\right\}, \quad \bar{\Gamma}^{(a)}\left(\overline{\mathbf{t}}^{(a)}\right):=\exp \left\{\sum_{\substack{n \geqslant 1, n \text { нечетне }}} B_{-n}^{(a)} \bar{t}_{n}^{(a)}\right\}, \\
B_{n}^{(a)}:=\frac{1}{2} \sum_{i \in \mathbb{Z}}(-1)^{i+1} \phi_{i}^{(a)} \phi_{-i-n}^{(a)} .
\end{gathered}
$$

Последовательность чисел $A=\left\{A_{n m}^{a, b}, a, b=1,2 ; n, m \in \mathbb{Z}\right\}$ представляет собой данные, определяющие тау-функцию двухкомпонентной иерархии 2В-КП. Двойка перед обозначением В-КП означает, что мы рассматриваем сдвоенную иерархию, что подразумевает, что тау-функция зависит как от левых, так и от правых наборов времен, обозначаемых соответственно $\mathbf{t}^{(a)} \overline{\mathbf{t}}^{(a)}$, в то время как индекс $a=1,2$ помечает номер компоненты времени. Четыре набора независимых параметров, $\mathbf{t}^{(a)}=\left(t_{1}^{(a)}, t_{3}^{(a)}, \ldots\right), \overline{\mathbf{t}}^{(a)}=\left(\bar{t}_{1}^{(a)}, \bar{t}_{3}^{(a)}, \ldots\right), a=1,2$, называются высшими временами иерархии. Если зафиксировать любые три набора, то четвертый будет представлять собой высшие времена обычной иерархии В-КП [7]. В этом смысле (19) можно рассматривать как тау-функцию четырех связанных копий иерархии В-КП. Если положить $\overline{\mathbf{t}}^{(1)}=\overline{\mathbf{t}}^{(2)}=0$, то мы получим двухкомпонентную иерархию В-КП, как это описано в работе [7].

В однокомпонентном случае мы опускаем вторую компоненту и верхние индексы. Тогда тау-функция иерархии 2B-КП принимает вид

$$
\tau^{2 \mathrm{~B}-\mathrm{K \Pi}}(\mathbf{t}, \overline{\mathbf{t}})=\left\langle 0\left|\Gamma(\mathbf{t}) \exp \left\{\sum_{n, m \in \mathbb{Z}} A_{n m} \phi_{n} \phi_{m}\right\} \bar{\Gamma}(\overline{\mathbf{t}})\right| 0\right\rangle,
$$


а тау-функцию обычной нейтральной иерархии В-КП можно записать как

$$
\tau^{\mathrm{B}-\mathrm{K \Pi}}(\mathbf{t})=\left\langle 0\left|\Gamma(\mathbf{t}) \exp \left\{\sum_{n, m \in \mathbb{Z}} A_{n m} \phi_{n} \phi_{m}\right\}\right| 0\right\rangle .
$$

Замечательный пример тау-функций иерархии В-КП был получен в работе [9], а именно $Q$-функции Шура $Q_{\alpha}$ [8]. Их можно выразить через фермионы:

$$
\left\langle 0\left|\Gamma(\mathbf{t}) \phi_{\alpha_{1}} \phi_{\alpha_{2}} \ldots \phi_{\alpha_{2 N}}\right| 0\right\rangle=2^{-\ell(\alpha) / 2} Q_{\alpha}\left(\frac{\mathbf{t}}{2}\right),
$$

где $\alpha_{1}>\alpha_{2}>\cdots>\alpha_{2 N} \geqslant 0$. Набор $\left(\alpha_{1}, \ldots, \alpha_{2 N}\right)$ и разбиение $\alpha$ связаны следующим образом: в случае $\alpha_{2 N}>0$ мы имеем $\alpha=\left(\alpha_{1}, \ldots, \alpha_{2 N}\right)$ и $\ell(\alpha)=2 N$, а в случае $\alpha_{2 N}=0$ мы имеем $\alpha=\left(\alpha_{1}, \ldots, \alpha_{2 N-1}\right)$ и $\ell(\alpha)=2 N-1$.

2.4. Фермионные представления для сумм. Приведенные выше формулы показывают, что суммы (1)-(6) являются тау-функциями иерархии В-КП. Прежде всего,

$$
\begin{aligned}
S_{3}\left(\mathbf{t}, A^{\mathrm{c}}\right) & =\sum_{\alpha \in D P} A_{\alpha}^{\mathrm{c}} Q_{\alpha}\left(\frac{\mathbf{t}}{2}\right)= \\
& =\left\langle 0\left|\Gamma(\mathbf{t}) \exp \left\{2 \sum_{n>m>0} A_{n m} \phi_{n} \phi_{m}+2 \sum_{n>0} a_{n} \phi_{n} \phi_{0}\right\}\right| 0\right\rangle .
\end{aligned}
$$

В соответствии с замечанием 1 мы получаем фермионные представления для сумм (1)-(4). В частности,

$$
\begin{aligned}
S_{0}(\mathbf{t}, L) & =\sum_{\substack{\alpha \in D P \\
\alpha 1 \leqslant L}} Q_{\alpha}\left(\frac{\mathbf{t}}{2}\right)=\left\langle 0\left|\Gamma(\mathbf{t}) \exp \left\{2 \sum_{L \geqslant n>m \geqslant 0} \phi_{n} \phi_{m}\right\}\right| 0\right\rangle \\
S_{1}\left(\mathbf{t}, \mathbf{t}^{*}\right) & =\sum_{\alpha \in D P} e^{-U_{\alpha}\left(\mathbf{t}^{*}\right)} Q_{\alpha}\left(\frac{\mathbf{t}}{2}\right)= \\
& =\left\langle 0\left|\Gamma(\mathbf{t}) \mathbb{T}\left(\mathbf{t}^{*}\right) \exp \left\{2 \sum_{n>m \geqslant 0} \phi_{n} \phi_{m}\right\}\right| 0\right\rangle \\
S_{4}\left(\mathbf{t}, \mathbf{t}^{*}\right) & =\sum_{\alpha \in D P^{2}} e^{-U_{\alpha}\left(\mathbf{t}^{*}\right)} Q_{\alpha}\left(\frac{\mathbf{t}}{2}\right)= \\
& =\left\langle 0\left|\Gamma(\mathbf{t}) \mathbb{T}\left(\mathbf{t}^{*}\right) \exp \left\{2 \sum_{n>0} \phi_{n} \phi_{n+1}\right\}\right| 0\right\rangle
\end{aligned}
$$

где

$$
\mathbb{T}\left(\mathbf{t}^{*}\right):=\exp \left\{-\sum_{n>0}(-1)^{n} U_{n}\left(\mathbf{t}^{*}\right) \phi_{n} \phi_{-n}\right\} .
$$

Вычисление при $\mathbf{t}=\mathbf{t}_{\infty}$ дает "солитонное" представление для $S_{1}\left(\mathbf{t}_{\infty}, \mathbf{t}^{*}\right)$ :

$$
\begin{aligned}
& S_{1}\left(\mathbf{t}_{\infty}, \mathbf{t}^{*}\right)= \\
& \quad=\frac{1}{c}\left\langle 0\left|\Gamma\left(\mathbf{t}_{+}^{*}\right) \exp \left\{2 \sum_{n>m>0} e^{-U_{m}^{(0)}-U_{n}^{(0)}} \phi(m)+2 \sum_{n>0} e^{-U_{n}^{(0)}} \phi(n) \phi_{0}\right\} \Gamma\left(\mathbf{t}_{-}^{*}\right)\right| 0\right\rangle
\end{aligned}
$$


где $\phi(n)$ обозначает фермионное поле (17) (доказательство см. в работе [14]). Это показывает, что данный ряд есть тау-функция иерархии 2В-КП относительно высших времен $\mathbf{t}_{ \pm}^{*}$, определяемых как $\mathbf{t}_{+}^{*}:=\left(t_{1}^{*}, t_{3}^{*}, \ldots\right)$ и $\mathbf{t}_{-}^{*}:=\left(-t_{-1}^{*},-t_{-3}^{*}, \ldots\right)$. Здесь $c-$ нормировочный множитель, задаваемый соотношением $\left\langle 0\left|\Gamma\left(\mathbf{t}_{+}^{*}\right) \Gamma\left(\mathbf{t}_{-}^{*}\right)\right| 0\right\rangle=b\left(\mathbf{t}_{-}^{*}, \mathbf{t}_{+}^{*}\right)$.

ЗАмЕчАниЕ 3. Еще одно фермионное представление для (3), (4) было получено в работе [13].

Наконец, получаем

$$
S_{5}\left(\mathbf{t}^{(1)}, \mathbf{t}^{(2)}, D\right)=\left\langle 0\left|\Gamma^{(1)}\left(\mathbf{t}^{(1)}\right) \Gamma^{(2)}\left(\mathbf{t}^{(2)}\right) \exp \left\{2 \sum_{n, m>0} \phi_{n}^{(1)} \phi_{m}^{(2)}\right\}\right| 0\right\rangle .
$$

\section{3. КРАТНЫЕ ИНТЕГРАЛЫ}

Пусть $d \nu$ - мера с носителем на контуре $\gamma$ на комплексной плоскости. Выберем в качестве $\gamma$ один их следующих двух контуров: интервал $0 \leqslant z<\infty$ на вещественной оси или дуга единичной окружности, задаваемая как $z=e^{i \varphi}, 0 \leqslant \varphi \leqslant \theta$, где $0<\theta<\pi$.

Рассмотрим следующие $N$-кратные интегралы:

$$
\begin{aligned}
I_{1}(N):=\int_{\gamma} \ldots \int_{\gamma}\left|\Delta^{*}(z)\right| \prod_{i=1}^{N} d \nu\left(z_{i}\right), & I_{2}(N):=\int_{\gamma} \ldots \int_{\gamma}\left|\Delta^{*}(z)\right|^{2} \prod_{i=1}^{N} d \nu\left(z_{i}\right), \\
I_{3}(N):=\int_{\gamma} \ldots \int_{\gamma} \Delta^{*}(z) a^{\mathrm{c}}(\mathbf{z}) \prod_{i=1}^{N} d \nu\left(z_{i}\right), & I_{4}(N):=\int_{\gamma} \ldots \int_{\gamma}\left|\Delta^{*}(z)\right|^{4} \prod_{i=1}^{N} d \nu\left(z_{i}\right),
\end{aligned}
$$

где, как и ранее,

$$
\Delta^{*}(z)=\prod_{i>j}^{N} \frac{z_{i}-z_{j}}{z_{i}+z_{j}}
$$

Аналогично (9) через $a^{\mathrm{c}}(\mathbf{z})$ обозначен пфаффиан $\operatorname{Pf}[\tilde{a}]$ антисимметричной матрицы $\tilde{a}$, элементы которой определяются в зависимости от четности $N$ в терминах кососимметричного ядра $a(z, w)$ (возможно, распределения) и функции (или распределения) $a(z)$ следующим образом: для четных $N=2 n$

$$
\tilde{a}_{i j}=-\tilde{a}_{j i}:=a\left(z_{i}, z_{j}\right), \quad 1 \leqslant i<j \leqslant 2 n ;
$$

для нечетных $N=2 n-1$

$$
\tilde{a}_{i j}=-\tilde{a}_{j i}:= \begin{cases}a\left(z_{i}, z_{j}\right), & \text { если } 1 \leqslant i<j \leqslant 2 n-1, \\ a\left(z_{i}\right), & \text { если } 1 \leqslant i<j=2 n .\end{cases}
$$

Кроме того, положим $a_{0}^{\mathrm{c}}=1$.

Интегралы $I_{1}, I_{2}$ и $I_{4}$ можно рассматривать как аналоги бета-ансамблей при $\beta=1,2,4$ [4]. Их можно получить как частные случаи $I_{3}$ следующим образом. Интеграл $I_{1}(N)$ является частным случаем $I_{3}(N)$, где в случае контура $\gamma$, заданного как интервал $0 \leqslant z<\infty$, следует положить $a\left(z_{i}, z_{j}\right)=\operatorname{sgn}\left(z_{i}-z_{j}\right)$ и $a(z)=1$; в случае контура $\gamma$, заданного как дуга единичной окружности $z=e^{i \varphi}, 0 \leqslant \varphi \leqslant \theta$, следует взять $a\left(z_{k}, z_{j}\right)=e^{-\pi i / 2} \operatorname{sgn}\left(\varphi_{k}-\varphi_{j}\right)$ и $a(z)=e^{-\pi i / 4}$, где $\varphi_{i}=\arg z_{i}$. Чтобы доказать это, можно воспользоваться следующим утверждением. 
Лемма. Справедливы равенства

$$
\begin{aligned}
\operatorname{Pf}\left[\operatorname{sgn}\left(z_{k}-z_{j}\right)\right] & =\operatorname{sgn} \Delta^{*}(z), \quad z_{k} \in \mathbb{R}, \\
\operatorname{Pf}\left[\operatorname{sgn}\left(\varphi_{k}-\varphi_{j}\right)\right] & =\operatorname{sgn}\left(e^{-\pi i\left(N^{2}-N\right) / 4} \Delta^{*}(z)\right), \quad z_{k}=e^{i \varphi_{k}},
\end{aligned}
$$

əде $k, j=1, \ldots, N$.

Интеграл $I_{2}(N)$ получается из $I_{3}(N)$, если положить $a\left(z_{i}, z_{j}\right)=\left(z_{i}-z_{j}\right) /\left(z_{i}+z_{j}\right)$ и $a(z)=1$. В этом случае мы пользуемся тем фактом, что

$$
\Delta^{*}(z)=\operatorname{Pf}\left[\frac{z_{i}-z_{j}}{z_{i}+z_{j}}\right]
$$

Интеграл $I_{4}(N)$ получается из $I_{3}(2 N)$ следующим образом. В случае контура $\gamma$, заданного как интервал $0 \leqslant z<\infty$, полагаем

$$
a\left(z_{i}, z_{j}\right)=\frac{1}{2}\left(z_{j} \frac{\partial}{\partial z_{j}} \delta\left(z_{i}-z_{j}\right)-\left(z_{i} \leftrightarrow z_{j}\right)\right)
$$

а в случае контура $\gamma$, заданного как дуга единичной окружности $z=e^{i \varphi}, 0 \leqslant \varphi \leqslant \theta$, полагаем

$$
a\left(z_{i}, z_{j}\right)=\frac{\partial}{\partial \varphi_{j}} \delta\left(\varphi_{i}-\varphi_{j}\right)
$$

Интегралы, содержащие $\Delta^{*}$, можно сравнить с интегралами, определяющими функции разбиения так называемых суперсимметричных матричных интегралов [19]. Интеграл $I_{2}$ определяет статистическую сумму так называемой модели $\widehat{A}_{0}$ [20], модели кулоновского газа с отражением [21], одномерной модели Изинга [22] и корреляционные функции в двумерной модели Изинга [23].

Чтобы связать данные интегралы с иерархией 2B-КП, мы введем деформации $I_{i}(N) \mapsto I_{i}(N ; \mathbf{t}, \overline{\mathbf{t}})$ посредством следующей деформации меры:

$$
d \nu(z) \mapsto d \nu(z \mid \mathbf{t}, \overline{\mathbf{t}})=b(\mathbf{t},\{z\}) b\left(-\overline{\mathbf{t}},\left\{z^{-1}\right\}\right) d \nu(z),
$$

где

$$
b(\mathbf{s}, \mathbf{t})=\exp \left\{\sum_{n \text { нечетное }} \frac{n}{2} s_{n} t_{n}\right\}, \quad\{z\}=\left(2 z, \frac{2 z^{3}}{3}, \frac{2 z^{5}}{5}, \ldots\right) .
$$

Ниже будет показано, что производящий ряд, полученный путем пуассонизации (статистическая сумма большого канонического ансамбля)

$$
Z_{i}(\mu ; \mathbf{t}, \overline{\mathbf{t}})=b(\mathbf{t}, \overline{\mathbf{t}}) \sum_{N=0}^{\infty} I_{i}(N ; \mathbf{t}, \overline{\mathbf{t}}) \frac{\mu^{N}}{N !}, \quad i=1,2,3,4,
$$

представляет собой частный случай тау-функций (20) иерархии 2B-КП.

Рассмотрим также следующие $2 N$-кратные интегралы:

$$
I_{5}\left(N ; \mathbf{t}^{(1)}, \mathbf{t}^{(2)}, \overline{\mathbf{t}}^{(1)}, \overline{\mathbf{t}}^{(2)}\right):=\int \Delta_{N}^{*}(z) \Delta_{N}^{*}(y) \prod_{i=1}^{N} d \nu\left(z_{i}, y_{i} \mid \mathbf{t}^{(1)}, \mathbf{t}^{(2)}, \overline{\mathbf{t}}^{(1)}, \overline{\mathbf{t}}^{(2)}\right)
$$


где

$$
\begin{aligned}
& d \nu\left(z, y \mid \mathbf{t}^{(1)}, \mathbf{t}^{(2)}, \overline{\mathbf{t}}^{(1)}, \overline{\mathbf{t}}^{(2)}\right)= \\
& \quad=b\left(\mathbf{t}^{(1)},\{z\}\right) b\left(-\overline{\mathbf{t}}^{(1)},\left\{z^{-1}\right\}\right) b\left(\mathbf{t}^{(2)},\{y\}\right) b\left(-\overline{\mathbf{t}}^{(2)},\left\{y^{-1}\right\}\right) d \nu(z, y)
\end{aligned}
$$

(здесь $d \nu(z, y)$ - произвольная бимера), и покажем, что производящий ряд

$$
Z_{5}\left(\mu ; \mathbf{t}^{(1)}, \mathbf{t}^{(2)}, \overline{\mathbf{t}}^{(1)}, \overline{\mathbf{t}}^{(2)}\right)=b\left(\mathbf{t}^{(1)}, \overline{\mathbf{t}}^{(1)}\right) b\left(\mathbf{t}^{(2)}, \overline{\mathbf{t}}^{(2)}\right) \sum_{N=0}^{\infty} I_{5}\left(N ; \mathbf{t}^{(1)}, \mathbf{t}^{(2)}, \overline{\mathbf{t}}^{(1)}, \overline{\mathbf{t}}^{(2)}\right) \frac{\mu^{N}}{N !}
$$

является частным случаем тау-функции (19) двухкомпонентной иерархии 2B-КП.

ЗАмечАниЕ 4. Если

$$
d \nu(z, y)=\delta(z-y) d \nu(z) d \nu(y), \quad \mathbf{t}=\mathbf{t}^{(1)}+\mathbf{t}^{(2)}, \quad \overline{\mathbf{t}}=\overline{\mathbf{t}}^{(1)}+\overline{\mathbf{t}}^{(2)},
$$

Tо

$$
Z_{2}(\mu ; \mathbf{t}, \overline{\mathbf{t}})=Z_{5}\left(\mu ; \mathbf{t}^{(1)}, \mathbf{t}^{(2)}, \overline{\mathbf{t}}^{(1)}, \overline{\mathbf{t}}^{(2)}\right) .
$$

Интегралы $Z_{1}(\mu ; \mathbf{t}, \overline{\mathbf{t}}), Z_{2}(\mu ; \mathbf{t}, \overline{\mathbf{t}}), Z_{4}(\mu ; \mathbf{t}, \overline{\mathbf{t}})$ и $Z_{5}\left(\mu ; \mathbf{t}^{(1)}, \mathbf{t}^{(2)}, \overline{\mathbf{t}}^{(1)}, \overline{\mathbf{t}}^{(2)}\right)$ можно получить как пределы по непрерывности сумм $S_{1}\left(\mathbf{t}_{\infty}, \mathbf{t}^{*}\right), S_{2}\left(\mathbf{t}_{\infty}, \mathbf{t}_{\infty}, \mathbf{t}^{*}\right), S_{4}\left(\mathbf{t}_{\infty}, \mathbf{t}^{*}\right)$ и $S_{5}\left(\mathbf{t}_{\infty}, \mathbf{t}_{\infty}, \mathbf{t}^{*}\right)$ соответственно.

Фермионное представление интегралов. Чтобы получить фермионное представление для приведенных выше интегралов, применим соотношение (18). Разлагая экспоненты и применяя теорему Вика к каждому слагаемому в сумме, получаем

$$
\begin{aligned}
Z_{3}(\mu ; \mathbf{t}, \overline{\mathbf{t}})= & \langle 0| \Gamma(\mathbf{t}) \exp \left\{\mu^{2} \int_{\gamma} \int_{\gamma} a(z, y) \phi(z) \phi(y) d \nu(z) d \nu(y)\right\} \times \\
& \times \exp \left\{2 \mu \int_{\gamma} a(z) \phi(z) \phi_{0} d \nu(z)\right\} \bar{\Gamma}(\overline{\mathbf{t}})|0\rangle .
\end{aligned}
$$

Заметим, что выражение

$$
Z_{3}(0 ; \mathbf{t}, \overline{\mathbf{t}})=b(\mathbf{t}, \overline{\mathbf{t}})=\langle 0|\Gamma(\mathbf{t}) \bar{\Gamma}(\overline{\mathbf{t}})| 0\rangle
$$

было выписано выше в (28). В частности, получаем

$$
\begin{aligned}
Z_{1}(\mu ; \mathbf{t}, \overline{\mathbf{t}})= & \langle 0| \Gamma(\mathbf{t}) \exp \left\{\mu^{2} q^{2} \int_{\gamma} \int_{\gamma} \operatorname{sgn}(\varsigma(z)-\varsigma(y)) \phi(z) \phi(y) d \nu(z) d \nu(y)\right\} \times \\
& \times \exp \left\{2 \mu q \int_{\gamma} \phi(z) \phi_{0} d \nu(z)\right\} \bar{\Gamma}(\overline{\mathbf{t}})|0\rangle,
\end{aligned}
$$

где в случае, когда контуром интегрирования является $\mathbb{R}_{+}$, имеем $q=1$ и $(z)=z$, $z \in \gamma$; а в случае, когда контур интегрирования есть дуга $z=e^{i \varphi}, 0 \leqslant \varphi \leqslant \theta$, мы имеем $q=e^{-\pi i / 4}$ и $\varsigma(z)=\varphi$.

Аналогично получаем

$$
\begin{aligned}
Z_{2}(\mu ; \mathbf{t}, \overline{\mathbf{t}})= & \langle 0| \Gamma(\mathbf{t}) \exp \left\{\mu^{2} \int_{\gamma} \int_{\gamma} \frac{z-y}{z+y} \phi(z) \phi(y) d \nu(z) d \nu(y)\right\} \times \\
& \times \exp \left\{2 \mu \int_{\gamma} \phi(z) \phi_{0} d \nu(z)\right\} \bar{\Gamma}(\overline{\mathbf{t}})|0\rangle .
\end{aligned}
$$


В качестве конкретизации выражения (29), выбрав $a(z, w)$ аналогично $(26)$ и положив $a(z)=0$, имеем

$$
Z_{4}(\mu ; \mathbf{t}, \overline{\mathbf{t}})=\left\langle 0\left|\Gamma(\mathbf{t}) \exp \left\{4 \mu \int_{\gamma} z \frac{\partial \phi(z)}{\partial z} \phi(z) d \nu(z)\right\} \bar{\Gamma}(\overline{\mathbf{t}})\right| 0\right\rangle .
$$

Наконец, в терминах двухкомпонентных фермионов получаем

$$
\begin{aligned}
& Z_{5}\left(\mu ; \mathbf{t}^{(1)}, \mathbf{t}^{(2)}, \overline{\mathbf{t}}^{(1)}, \overline{\mathbf{t}}^{(2)}\right)=\frac{1}{c}\langle 0| \Gamma^{(1)}\left(\mathbf{t}^{(1)}\right) \Gamma^{(2)}\left(\mathbf{t}^{(2)}\right) \times \\
& \quad \times \exp \left\{2 \mu \iint \phi^{(1)}(z) \phi^{(2)}(y) d \nu(z, y)\right\} \bar{\Gamma}^{(1)}\left(\overline{\mathbf{t}}^{(1)}\right) \bar{\Gamma}^{(2)}\left(\overline{\mathbf{t}}^{(2)}\right)|0\rangle,
\end{aligned}
$$

где $c:=Z_{5}\left(0 ; \mathbf{t}^{(1)}, \mathbf{t}^{(2)}, \overline{\mathbf{t}}^{(1)}, \overline{\mathbf{t}}^{(2)}\right)-$ нормировочный множитель.

Формулы (29), (30), (31) и (32) нужно сравнить с формулами (21), (23), (24) и (25) соответственно. Фермионные представления интегралов $Z_{1}$ и $Z_{4}$ аналогичны результатам, полученным в работе [24] для ансамблей вещественных симметричных и самодуальных кватернионных случайных матриц. Фермионное представление (32) следует сравнить с фермионным представлением для статистической суммы двухматричной модели, полученным в работе [25].

\section{4. ОБСУЖДЕНИЕ}

Представлены пять типов кратных сумм и кратных интегралов, связаных с тауфункциями иерархий В-КП (с тау-функциями иерархии В-КП и двухкомпонентной иерархии В-КП для кратных сумм, с тау-функциями иерархии 2B-KП и двухкомпонентной иерархии 2В-КП для кратных интегралов). Известно, что некоторые из этих сумм и интегралов имеют применения в математике и физике; мы полагаем, что все их можно использовать в различных вероятностных моделях. Технику вычислений свободных фермионов и интегрируемых систем можно применить для изучения различных свойств этих сумм и интегралов. Тау-функции многокомпонентной иерархии В-КП [7] можно затем использовать для построения моделей пфаффиановых процессов (ср. [26]). Результаты настоящей работы следует сравнить с аналогичными результатами для случая так называемой заряженной иерархии В-КП [7], исследованного в работе [24]. Это также будет предметом наших дальнейших исследований.

\section{Применение интеграла $Z_{2}(N)$}

\section{ПРИЛОЖЕНИЕ}

Исследовать некоторые вопросы, рассмотренные в настоящей работе, нас побудили следующие наблюдения, сообщенные нам Г. Браденом [23].

Применение интегралов $Z_{2}$ относится к модели Изинга для корреляционной функции, когда имеется возмущение относительно критической температуры. В скейлинговом пределе это описывается системой майорановских фермионов и устанавливает связь с рассматриваемым фермионным представлением. Маккой, Трейси и Ву показали, что этот предел описывается теорией массивного $\left(m=T-T_{\mathrm{c}}\right)$ поля, корреляции которого подчиняются радиальному уравнению sh-Гордон, представляющему 
собой при замене параметров уравнение Пенлеве III. Другой подход к тому же коррелятору заключается в использовании формфактора. Этот подход дает коррелятор в терминах бесконечной суммы кратных интегралов. Для рассматриваемого случая мы имеем евклидову корреляционную функцию типа

$$
\begin{aligned}
G(r):=\langle\mathcal{O}(x) \mathcal{O}(0)\rangle & =\sum_{n=0}^{\infty} \int \frac{\prod_{i=1}^{n} d \beta_{i}}{n !(2 \pi)^{n}}\left\langle 0|\mathcal{O}(x)| \beta_{1} \ldots \beta_{n}\right\rangle\left\langle\beta_{n} \ldots \beta_{1}|\mathcal{O}(0)| 0\right\rangle= \\
& =\sum_{n=0}^{\infty} \int \frac{\prod_{i=1}^{n} d \beta_{i}}{n !(2 \pi)^{n}}\left|F_{n}\left(\beta_{1} \ldots \beta_{n}\right)\right|^{2} \exp \left(-m r \sum_{i=1}^{n} \operatorname{ch} \beta_{i}\right) .
\end{aligned}
$$

Здесь $\beta_{i}$ - быстроты, $x=\left(x_{0}, x_{1}\right), r=\sqrt{x_{0}^{2}+x_{1}^{2}}$. Если использовать минимальный формфактор

$$
F_{n}^{\min }\left(\beta_{1} \ldots \beta_{n}\right)=\prod_{i<j} \operatorname{th}\left(\frac{\beta_{i}-\beta_{j}}{2}\right)
$$

и подходящие нормировки, то получим

$$
G\left(\frac{r}{m}\right)=\sum_{n=0}^{\infty} \frac{1}{n !} \frac{1}{(2 \pi)^{n}} \int_{0}^{\infty} \prod_{i=1}^{n}\left(\frac{d x_{i}}{x_{i}} e^{-r\left(x_{i}+1 / x_{i}\right)}\right) \prod_{i<j}\left(\frac{x_{i}-x_{j}}{x_{i}+x_{j}}\right)^{2} .
$$

Поскольку коррелятор никак не конкретизирован, нас также интересует

$$
G_{ \pm}\left(\frac{r}{m}\right)=\sum_{n=0}^{\infty} \frac{1}{n !} \frac{( \pm 1)^{n}}{(2 \pi)^{n}} \int_{0}^{\infty} \prod_{i=1}^{n}\left(\frac{d x_{i}}{x_{i}} e^{-r\left(x_{i}+1 / x_{i}\right)}\right) \prod_{i<j}\left(\frac{x_{i}-x_{j}}{x_{i}+x_{j}}\right)^{2} .
$$

Тождество

$$
\operatorname{det}\left(\frac{1}{x_{i}+x_{j}}\right)=\frac{1}{2^{n} x_{1} \ldots x_{n}} \prod_{i<j}\left(\frac{x_{i}-x_{j}}{x_{i}+x_{j}}\right)^{2}
$$

дает связь с определителем Фредгольма. Трудность заключается в том, чтобы получить сходящееся разложение выражения (33). Первое слагаемое записывается в терминах $K_{0}(r)$. Такое разложение даст (частное) решение уравнения Пенлеве.

Благодарности. Авторы выражают благодарность Т. Шиоте и Дж. Дж. С. Ниммо за полезные обсуждения. А. Ю. Орлов благодарит А. Одзиевича за гостеприимство во время его пребывания в Белостоке в июне 2005 года. А. Ю. Орлов и Й. В. ван де Лёр благодарят Centre de recherches mathématiques, Université de Montréal (Montréal, Canada) за гостеприимство во время их пребывания там в январе 2006 года, когда была написана основная часть настоящей работы. Работа выполнена при частичной финансовой поддержке Евросоюза в рамках Программы FP6 Marie Curie RTN ENIGMA (контракт № MRTN-CT-2004-5652) и Программы MISGAM 8 Европейского научного фонда, а также Natural Sciences and Engineering Research Council of Canada и Fonds FCAR du Québec (Canada); поддержана Программой РAH "Фундаментальные проблемы нелинейной динамики" и РФФИ (гранты № 09-01-92437-KE_а и $08-01-00501)$. 


\section{Список литературы}

[1] A. Okounkov, "The uses of random partitions", XIVth International Congress on Mathematical Physics, eds. J.-C. Zambrini, World Sci., Hackensack, NJ, 2005, 379-403, arXiv: math-ph/0309015.

[2] M.E. Fisher, J. Stat. Phys., 34:5-6 (1984), 667-729.

[3] P. J. Forrester, Log-Gases and Random Matrices, London Math. Soc. Monogr. Ser., 34, Princeton Univ. Press, Princeton, NJ, 2010.

[4] M. L. Mehta, Random Matrices, Pure Appl. Math., 142, Elsevier, Amsterdam, 2004.

[5] E. Date, M. Jimbo, M. Kashiwara, T. Miwa, Physica D, 4:3 (1982), 343-365.

[6] M. Jimbo, T. Miwa, Publ. RIMS Kyoto Univ., 19:3 (1983), 943-1001.

[7] V.G. Kac, J.van de Leur, "The geometry of spinors and the multicomponent BKP and DKP hierarchies", The Bispectral Problem, CRM Proc. Lect. Notes, 14, eds. J. Harnad, A. Kasman, AMS, Providence, RI, 1998, 159-202.

[8] И. Макдональд, Симметрические функиии и многочлены Холла, Мир, М., 1985.

[9] Y. You, "Polynomial solutions of the BKP hierarchy and projective representations of symmetric groups", Infinite-dimensional Lie Algebras and Groups, Adv. Ser. Math. Phys., 7, eds. V. G. Kac, World Sci., Teaneck, NJ, 1989, 449-464.

[10] J. J. C. Nimmo, J. Phys. A, 23:5 (1990), 751-760.

[11] A. Yu. Orlov, T. Shiota, Phys. Lett. A, 343:5 (2005), 384-396, arXiv: math-ph/0501017.

[12] C. A. Tracy, H. Widom, Duke Math. J., 123:1 (2004), 171-208, arXiv: math.PR/0210255.

[13] А. Ю. Орлов, ТМФ, 137:2 (2003), 253-270, arXiv: math-ph/0302011.

[14] J. W. van de Leur, A. Yu. Orlov, Phys. Lett. A, 373:31 (2009), 2675-2681, arXiv: 0801.0066.

[15] A. Okounkov, "SL(2) and z-measures", Random Matrix Models and Their Applications, Math. Sci. Res. Inst. Publ., 40, eds. P. Bleher, A. Its, Cambridge Univ. Press, Cambridge, 2001, 407-420, arXiv: math.RT/0002135.

[16] O. Foda, M. Wheeler, M. Zuparic, J. Algebra, 321:11 (2009), 3249-3273, arXiv: 0808.2737.

[17] M. Vuletić, Int. Math. Res. Not., 2007:14 (2007), 043, 53 pp., arXiv: math-ph/0702068.

[18] Дж. Харнад, А. Ю. Орлов, ТМФ, 158:1 (2009), 23-48.

[19] T. Guhr, J. Math. Phys., 32:2 (1991), 336-347.

[20] I. K. Kostov, Nucl. Phys. B Proc. Suppl., 45:1 (1996), 13-28, arXiv: hep-th/9509124.

[21] I. Loutsenko, V. Spiridonov, J. Stat. Phys., 99:3-4 (2000), 751-767, arXiv: cond-mat/9909308.

[22] I. M. Loutsenko, V.P. Spiridonov, SIGMA, 3 (2007), 059, 11 pp., arXiv: 0704.3173.

[23] Г. Браден, частное сообщение, 2006.

[24] J. van de Leur, J. Nonlin. Math. Phys., 8:2 (2001), 288-311, arXiv: solv-int/9909028.

[25] J. Harnad, A. Yu. Orlov, J. Phys. A, 39:28 (2006), 8783-8809, arXiv: math-ph/0512056.

[26] Дж. Харнад, А. Ю. Орлов, ТМФ, 152:2 (2007), 265-277, arXiv: 0704.1145. 